\title{
Resource allocation and sucrose mobilization in light-limited eelgrass Zostera marina
}

\author{
Teresa Alcoverro ${ }^{1, *}$, Richard C. Zimmerman ${ }^{2}$, Donald G. Kohrs ${ }^{2}$, Randall S. Alberte ${ }^{3}$ \\ ${ }^{1}$ Centre d'Estudis Avançats de Blanes (CSIC), Carretera Sta. Bàrbara s/n, 17300 Blanes, Girona, Spain \\ ${ }^{2}$ Moss Landing Marine Laboratories, San Jose State University Foundation, PO Box 450, Moss Landing, California 95039, USA \\ ${ }^{3}$ Areté Associates Inc, 1725 Jefferson Davis Hwy, Arlington, Virginia 22202, USA
}

\begin{abstract}
This study evaluated the ability of Zostera marina L. (eelgrass) to balance the daily photosynthetic deficit by mobilization of carbon reserves stored in below-ground tissues during a period of extreme winter light limitation. A quantitative understanding of the mobilization process and its limitations is essential to the development of robust models predicting minimum light levels required to maintain healthy seagrass populations. Plants were grown in running seawater tanks under 2 light regimes. One treatment was provided with $2 \mathrm{~h}$ irradiance-saturated photosynthesis $\left(H_{\mathrm{sat}}\right)$ to produce severe light limitation, while control plants were grown under $7 \mathrm{~h} H_{\text {sat }}$ simulating the typical wintertime condition in Monterey Bay, California, USA. Although plants maintained under $2 \mathrm{~h} H_{\text {sat }}$ were more severely carbon limited than plants grown under $7 \mathrm{~h} H_{\text {sal }}$, whole-plant carbon balance calculated from metabolic needs and growth rates was negative for both $H_{3 a t}$ treatments. The eelgrass studied here responded to negative carbon balances by suppressing the production of new roots, depleting sucrose reserves, and effecting a gradual decrease in growth rate and an increase in the activity of sucrose synthase (SS, E.C. 2.4.1.13) in sink tissues in the terminal stages of carbon stress. The $7 \mathrm{~h} H_{\text {sat }}$ plants survived the $45 \mathrm{~d}$ course of the experiment while the plants grown under $2 \mathrm{~h} H_{\text {sat }}$ died within $30 \mathrm{~d}$, even though one-third of their carbon reserves remained immobilized in the rhizome. Thus, extreme light limitation can prevent full mobilization of carbon reserves stored in below-ground tissues, probably through the effects of anoxia on translocation. Metabolic rates, particularly photosynthesis and respiration of the shoot, were unaffected by prolonged carbon limitation in both treatments. The patterns observed here can provide useful indices for assessing the state and fate of seagrass ecosystems in advance of catastrophic declines.
\end{abstract}

KEY WORDS: Seagrass Carbon balance - Resource allocation - Photosynthesis - Light

\section{INTRODUCTION}

Resource limitation impacts the resulting growth form and pattern of resource allocation to above- and below-ground tissues of all plants. Although nutrient limitation frequently results in the proliferation of roots at the expense of above-ground growth, light or carbon limitation mobilizes stored reserves to support shoot or leaf proliferation at the expense of belowground growth (Pierson et al. 1990, Perez et al. 1994, Sims \& Pearcey 1994, Canham et al. 1996, Zimmerman

•E-mail: teresa@ceab.csic.es et al. 1996, 1997, Clabby \& Osborne 1997). Among marine macrophytes, seagrasses (marine angiosperms) are particularly vulnerable to light limitation, especially in temperate and subpolar waters (Backman \& Barilatti 1976, Dennison \& Alberte 1982, 1985, 1986, Pirc 1989, Tomasko \& Dawes 1989, Duarte 1991, Zimmerman et al. 1991, Dunton \& Tomasko 1994, Zimmerman et al. 1995b, Moore et al. 1997). Healthy eelgrass requires 5 to $6 \mathrm{~h}$ of irradiance-saturated photosynthesis $\left(H_{\text {sat }}\right)$ each day to maintain positive carbon balance and vigorous growth (Zimmerman et al. 1995b, 1996, Zimmerman \& Mobley 1997), although external factors, including leaf grazing from the commensal limpet Tectura depicta (Berry), can significantly increase that 
light requirement (Zimmerman et al. 1996). Given that winter light levels in temperate latitudes may hover near or below the $6 \mathrm{~h} H_{\text {sat }}$ threshold during winter months (McRoy 1969, Carruthers \& Walker 1997), seagrass survival may depend on the utilization of carbon reserves accumulated during summer. The effects of light limitation on resource allocation and reserve mobilization in seagrasses, however, are poorly understood.

Periods of light-limited photosynthesis may change carbon allocation strategies which can significantly impact eelgrass growth and survival. Roots are critical for nutrient acquisition and stabilization of seagrass shoots within unconsolidated sediments (Harlin \& Throne-Miller 1981, Iizumi \& Hattori 1982, Zimmerman et al. 1987), but the maintenance of healthy roots in permanently flooded anoxic sediments depends on photosynthetically derived oxygen for daytime aerobiosis and sufficient reserves of reduced carbon to support anaerobic metabolism at night when translocation is blocked by anoxia (Smith 1989, Zimmerman \& Alberte 1996). Winter is a period of maximum turbidity in water columns of temperate estuaries and nearshore environs. Storm-driven sediment loading combines with short daylengths to generate periods of extreme light limitation which prevent translocation of reduced carbon from eelgrass leaves to the roots and rhizomes and may prevent the mobilization of reserves accumulated in below-ground tissues during the summer period of high light availability (Pennock \& Sharp 1994, Zimmerman et al. 1994, 1995b, Hillman et al. 1995, Zimmerman \& Alberte 1996, Fetweis et al. 1998).

The objective of this study was to evaluate the ability of Zostera marina L. (eelgrass) to balance the daily photosynthetic deficit by mobilizing carbon reserves stored in below-ground tissues during a period of extreme winter light limitation. A quantitative understanding of the mobilization process and its limitations

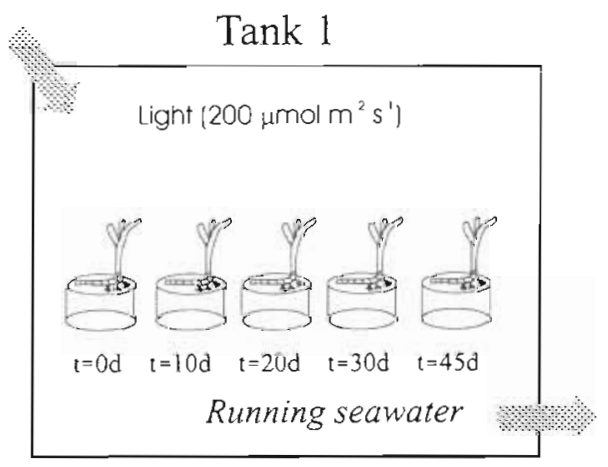

Fig. 1. Experimental design of 1 tank with running seawater and the 5 cores for each sampling time. There were 5 tanks per treatment ( 5 replicates) and 2 treatments $(2 \mathrm{~h}$ and $7 \mathrm{~h}$ ) is essential to the development of robust models predicting minimum light levels required to maintain healthy seagrass populations, as reliance on simple carbon balance arguments may seriously overestimate the potential for seagrasses to survive periods of extreme light limitation, particularly in temporally variable habitats.

\section{METHODS}

Experimental design. In total, 50 Zostera marina plants growing at the deep edge (10 $\mathrm{m}$ depth) of an eelgrass meadow near Del Monte Beach, Monterey Bay, California $\left(36^{\circ} 30^{\prime} 40^{\prime \prime} \mathrm{N}, 121^{\circ} 52^{\prime} 30^{\prime \prime} \mathrm{W}\right)$ were collected with SCUBA in late November 1993 using the rhizosphere core method (Dennison \& Alberte 1982) and transported to the laboratory within $2 \mathrm{~h}$ of collec. tion. Each core contained an intact single shoot with 10 to 12 rhizome internodes and associated roots with intact sediment. The leaf grazing limpet Tectura depicta was present on all plants collected, and their numbers were not otherwise manipulated as part of this experiment. Five separate cores were placed into each of the ten $150 \mathrm{l}$ tanks plumbed with running seawater flowing at 3 turnovers $h^{-1}$ at a temperature of $12^{\circ} \mathrm{C}$ (Fig. 1). Illumination was provided from above by timer-activated $300 \mathrm{~W}$ quartz-halogen lamps producing $200 \mu \mathrm{mol}$ quanta $\mathrm{m}^{-2} \mathrm{~s}^{-1}$ of photosynthetically active radiation (PAR) to the bottom of each tank, well above the photosynthesis saturating irradiance $\left(E_{k}\right)$ of 30 to $50 \mu \mathrm{mol}$ quanta $\mathrm{m}^{-2} \mathrm{~s}^{-1}$ required for plants growing in situ during December 1993 and January 1994 (Zimmerman unpubl. data). A total of 25 cores distributed among 5 replicate tanks were illuminated for only $2 \mathrm{~h}$ each day $\left(H_{\text {sat }}=2 \mathrm{~h}\right)$ to induce severe light limitation, while the other 5 replicate tanks (25 cores) received $7 \mathrm{~h}$ of illumination to simulate the mean daily $H_{\text {sat }}$ period recorded at the collection site during December 1993 and January 1994 (Zimmerman unpubl. data)

One core was harvested from each replicate tank every 10 d ( 5 samples per $H_{\text {sat }}$ treatment) and analyzed for biomass distribution (shoots, rhizomes and roots), number of internodes, growth rate, maximum net photosynthesis $\left(P_{\mathrm{m}}\right)$ and respiration $(R)$, the activity of sucrose-mobilizing enzymes sucrose phosphate synthase (SPS, E.C. 2.4.1.14) in leaves and sucrose synthase (SS, E.C. 2.4.1.13) in roots, protein content and total carbohydrate reserves (TCR).

Metabolic rates and leaf chlorophyll. Leaf $P_{m}$ and leaf, root and rhizome $R$ were measured at ambient growth temperature $\left(15^{\circ} \mathrm{C}\right)$ using temperature-controlled polarographic $\mathrm{O}_{2}$ electrodes. Respiration rates of rhizomes (first [= youngest] internode) and roots 
(youngest bundle on each rhizome) were measured in $\mathrm{O}_{2}$-enriched seawater to simulate the lacunal $\mathrm{O}_{2}$ tension generated by fully illuminated intact plants and to prevent $\mathrm{O}_{2}$ limitation of respiration (Zimmerman et al. 1989). Leaf $P_{\mathrm{m}}$ and $R$ were measured during $20 \mathrm{~min}$ around noon on $2 \mathrm{~cm}$ segments removed from the middle portion of Leaves 2, 3 and 4. Illumination (PPF = $500 \mu \mathrm{mol}$ photons $\mathrm{m}^{-2} \mathrm{~s}^{-1}$ ) was provided by $35 \mathrm{~mm}$ slide projectors. Leaf respiration was measured in the dark using air-saturated seawater. The newly emerged leaf (1) was generally too small to sample, and the oldest leaf (5), if present, was generally senescent. After metabolic rate determinations, each leaf segment was ground in cold $90 \%(\mathrm{v} / \mathrm{v})$ acetone for spectrophotometric determination of total chlorophyll $(a+b)$ content using the extinction coefficients of Jeffrey \& Humphrey (1975).

The ability to estimate whole-plant metabolic activity from the middle segments of Leaves 2, 3 and 4 was compared to a more detailed estimate based on measurements of metabolic rates at 4 positions on each leaf. Five shoots were collected from Del Monte Beach, Monterey Bay in January 1994. Segments were cut from the sheath (that portion that still sequestered with the leaf sheath), base (the youngest emergent third of each leaf), middle (middle one-third), and tip (upper one-third) of each leaf, and metabolic rates measured polarographically as above. Metabolic rates of individual leaves were then calculated by integrating the measured rates of these compartmentalized segments relative to their proportion of biomass in each leaf. Metabolic rates of individual shoots were calculated by scaling the integrated rates of Leaves 2, 3 and 4 according to their biomass distribution in the shoot. These estimates were then contrasted with integrals calculated using only the middle segments of each leaf.

Enzyme activity. The capacity for sucrose formation and export from leaves was evaluated by measuring the maximum velocity ( $\left.V_{\max }\right)$ activity of SPS in crude extracts (Zimmerman et al. 1995a). Sink strength of translocation-dependent roots, as measured by SS activity, was assayed in the youngest root bundle emerging from each shoot (Zimmerman et al. 1995a). Protein content of the enzyme extract was determined by dot-blot analysis using a dye-binding assay (Winterbourne 1986).

Total carbohydrate reserves. Sugars were extracted from ground samples of leaf, root and rhizome in hot $\left(80^{\circ} \mathrm{C}\right.$ ) ethanol (Zimmerman et al. 1989). The extracts were evaporated to dryness at room temperature under a stream of compressed air, redissolved in distilled water and analyzed spectrophotometrically using a resorcinol assay standardized to sucrose (Huber \& Israel 1982). Starch was extracted from ethanol-insoluble residue overnight in $1 \mathrm{~N} \mathrm{KOH}$ and analyzed spec- trophotometrically using an anthrone assay standardized to sucrose (Yemn \& Willis 1954). Sucrose and starch contents were added to obtain the TCR.

Growth rates and biomass allocation. Five days prior to harvest, shoots were marked above the meristem with a hypodermic needle and left to grow. Growth was determined on the harvest day by measuring the total length of each leaf and the length of new tissue below the punch mark on each leaf (Zieman 1974, Zimmerman et al. 1996). Young leaves without punch marks were assumed to have been produced entirely after marking. Biomass-specific growth was calculated by normalizing the length of new leaf tissue (below the punch marks) to the total length of all leaves on each shoot. In addition, fresh weight of leaves, rhizomes, roots and rhizome lengths (number of internodes) were measured. Growth of subterranean tissue was not measured but was assumed proportional to leaf growth rates for calculating carbon budgets.

Statistical analyses. Statistical significance of treatment effects were determined by 2-way ANOVA ( $H_{\text {sat }}$ $\times$ Time) or 3-way ANOVA ( $H_{\text {sat }} \times$ Time $\times$ Tissue) for each variable measured. Only observations to $30 \mathrm{~d}$ were included in the analysis because none of the plants grown under $2 \mathrm{~h} H_{\text {sat }}$ periods survived to $45 \mathrm{~d}$.

Whole plant carbon balance. Measured rates of $P_{\mathrm{m}}$, $R$, and growth were used to calculate daily carbon balances. Metabolic rates were converted from units of $\mathrm{O}_{2}$ to $\mathrm{C}$ using molar photosynthetic and respiratory quotients of $1.0\left(\mathrm{O}_{2}: \mathrm{CO}_{2}\right)$. Daily net carbon gain (or loss) in the shoot was then calculated according to Zimmerman et al. (1996) as:

$$
C_{\text {gain }}=\left[P_{\mathrm{m}} \times H_{\text {sat }}\right]-\left[R_{\mathrm{s}}\left(24-H_{\text {sat }}\right)\right]
$$

where $P_{m}$ was the light-saturated rate of net photosynthesis, $R_{\mathrm{s}}$ was the rate of dark respiration of the photosynthetic shoot, and $H_{\text {sat }}$ was either 2 or $7 \mathrm{~h}$. Aggregate metabolic rates of shoots for each sampling period were obtained by scaling the measured rates of $P_{\mathrm{m}}$ and $R_{\mathrm{s}}$ of the middle segments of Leaves 2,3 and 4 to the relative biomass of those leaves in each shoot.

Carbon demand of root and rhizome $\left(D_{R-R}\right)$ Internode 1 was calculated from measured rates of respiration. For the remaining internodes, $D_{\text {R-R }}$ was obtained from the exponential relationship reported by Kraemer \& Alberte (1993). The rate of carbon consumption by below-ground tissues during the dark (anaerobic) period was assumed to be $65 \%$ of the rate during the light (aerobic) period, as shown by Smith (1989):

$$
D_{\mathrm{R}-\mathrm{R}}=\left[R \times H_{\text {sat }}\right]+\left[0.65 \times R \times\left(24-H_{\text {sat }}\right)\right]
$$

where $R$ was the respiration rate of roots and rhizomes measured at rate-saturating $\mathrm{O}_{2}$ tensions (200\% of air saturation). The biomass-specific rates were then 
Table 1 Results of 3 -way ANOVA examining the effects of Time, $H_{\text {sat }}$ and leaf age on photosynthesis, respiration, photosynthesis:respiration (P:R) and chlorophyll $(a+b)$ content. df: degrees of freedom for each treatment, MS: mean-square values, $F$ : resulting F-ratio, p: probability that the independent variables had no effect on the dependent variables. (") Treatments effects were considered statistically significant if $p<0.05 .(\cdots) p<0.001$. ns: not significant

\begin{tabular}{|c|c|c|c|c|c|c|}
\hline Dependent variable & Independent variable & $\mathrm{df}$ & MS & $F$ & $\mathrm{p}$ & Significance \\
\hline $\begin{array}{l}\text { Photosynthesis } \\
\left(\mu \mathrm{mol} \mathrm{O} \mathrm{g}^{-1} \mathrm{FW} \mathrm{min}^{-1}\right)\end{array}$ & $\begin{array}{l}\text { Time } \\
\text { Leaf age } \\
H_{\text {sat }} \\
\text { Time } \times \text { Leaf age } \\
\text { Time } \times H_{\text {sat }} \\
\text { Leaf age } \times H_{\text {sat }} \\
\text { Time } \times \text { Leaf age } \times H_{\text {sdt }} \\
\text { Within }\end{array}$ & $\begin{array}{l}3 \\
2 \\
1 \\
6 \\
3 \\
2 \\
6 \\
96\end{array}$ & $\begin{array}{l}0.009 \\
0.234 \\
0.013 \\
0.016 \\
0.022 \\
0.004 \\
0.006 \\
0.010\end{array}$ & $\begin{array}{r}0.96 \\
24.42 \\
1.34 \\
1.67 \\
2.34 \\
0.45 \\
0.66\end{array}$ & $\begin{array}{r}0.414 \\
<0.001 \\
0.249 \\
0.136 \\
0.078 \\
0.640 \\
0.683\end{array}$ & $\begin{array}{l}\text { ns } \\
\text { ns } \\
\text { ns } \\
\text { ns } \\
\text { ns } \\
\text { ns }\end{array}$ \\
\hline $\begin{array}{l}\text { Respiration } \\
\left(\mu \mathrm{mol} \mathrm{O} \mathrm{O}_{2} \mathrm{~g}^{-1} \mathrm{FW} \mathrm{min}^{-1}\right)\end{array}$ & $\begin{array}{l}\text { Time } \\
\text { Leaf age } \\
H_{\text {sdt }} \\
\text { Time } \times \text { Leaf age } \\
\text { Time } \times H_{\text {sat }} \\
\text { Leaf age } \times H_{\text {sat }} \\
\text { Time } \times \text { Leaf age } \times H_{\text {sat }} \\
\text { Within }\end{array}$ & $\begin{array}{r}3 \\
2 \\
1 \\
6 \\
3 \\
2 \\
6 \\
96\end{array}$ & $\begin{array}{l}0.003 \\
0.008 \\
0.008 \\
0.006 \\
0.007 \\
0.003 \\
0.002 \\
0.003\end{array}$ & $\begin{array}{l}1.13 \\
2.71 \\
2.74 \\
2.04 \\
2.65 \\
1.02 \\
0.83\end{array}$ & $\begin{array}{l}0.341 \\
0.072 \\
0.101 \\
0.068 \\
0.053 \\
0.366 \\
0.547\end{array}$ & $\begin{array}{l}\text { ns } \\
\text { ns } \\
\text { ns } \\
\text { ns } \\
\text { ns } \\
\text { ns } \\
\text { ns }\end{array}$ \\
\hline$P: R$ & $\begin{array}{l}\text { Time } \\
\text { Leaf age } \\
H_{\text {sat }} \\
\text { Time } \times \text { Leaf age } \\
\text { Time } \times H_{\text {sat }} \\
\text { Leaf age } \times H_{\text {sat }} \\
\text { Time } \times \text { Leaf age } \times H_{\text {sat }} \\
\text { Within }\end{array}$ & $\begin{array}{r}3 \\
2 \\
1 \\
6 \\
3 \\
2 \\
6 \\
93\end{array}$ & $\begin{array}{r}9.19 \\
27.65 \\
3.44 \\
4.18 \\
0.76 \\
0.95 \\
0.92 \\
3.01\end{array}$ & $\begin{array}{l}3.05 \\
9.18 \\
1.14 \\
1.38 \\
0.25 \\
0.31 \\
0.30\end{array}$ & $\begin{array}{r}0.032 \\
<0.001 \\
0.287 \\
0.227 \\
0.858 \\
0.729 \\
0.932\end{array}$ & $\begin{array}{l}\cdots \\
\text { ns } \\
\text { ns } \\
\text { ns } \\
\text { ns } \\
\text { ns }\end{array}$ \\
\hline $\begin{array}{l}\text { Chlorophyll }(a+b) \\
\left(\mathrm{mg} \text { chl g }{ }^{-1} \mathrm{FW}\right)\end{array}$ & $\begin{array}{l}\text { Time } \\
\text { Leaf age } \\
H_{\text {sat }} \\
\text { Time } \times \text { Leaf age } \\
\text { Time } \times H_{\text {sat }} \\
\text { Leaf age } \times H_{\text {sat }} \\
\text { Time } \times \text { Leaf age } \times H_{\text {sat }} \\
\text { Within }\end{array}$ & $\begin{array}{r}3 \\
2 \\
1 \\
6 \\
3 \\
2 \\
6 \\
89\end{array}$ & $\begin{array}{l}7.473 \\
0.027 \\
0.179 \\
0.111 \\
0.038 \\
0.035 \\
0.023 \\
0.070\end{array}$ & $\begin{array}{r}106.1 \\
0.38 \\
2.54 \\
1.57 \\
0.53 \\
0.49 \\
0.33\end{array}$ & $\begin{array}{r}<0.001 \\
0.679 \\
0.115 \\
0.165 \\
0.659 \\
0.612 \\
0.919\end{array}$ & $\begin{array}{l}\cdots \\
\text { ns } \\
\text { ns } \\
\text { ns } \\
\text { ns } \\
\text { ns } \\
\text { ns }\end{array}$ \\
\hline
\end{tabular}

Table 2. Average of $P_{\mathrm{m}}$ respiration, protein content and biomass for the different tissues. Standard deviations are given in brackets

\begin{tabular}{|c|c|c|c|c|c|}
\hline & Leaf 2 & Leaf 3 & Leaf 4 & Rhizome & Root \\
\hline Biomass (g FW) & & & & $3.48(1.30)$ & $1.53(0.85)$ \\
\hline $\begin{array}{l}\text { Maximum net photosynthesis } \\
\left.\left(\mu \mathrm{mol} \mathrm{O}_{2} g^{-1} \mathrm{FW} \mathrm{min}\right)^{-1}\right)\end{array}$ & $0.301(0.122)$ & $0.229(0.093)$ & $0.145(0.083)$ & & \\
\hline$P_{\mathrm{q}}: R$ & $3.71(2.16)$ & $3.50(2.21)$ & $2.39(1.57)$ & & \\
\hline Respiration ( $\mu$ mol $\mathrm{O}_{2} \mathrm{~g}^{-1} \mathrm{FW} \mathrm{min}{ }^{-1}$ ) & $-0.099(0.053)$ & $-0.088(0.059)$ & $-0.067(0.051)$ & $-0.031(0.022)$ & $-0.038(0.037)$ \\
\hline Protein (mg g ${ }^{-1}$ FW $)$ & & $10.74(3.81)$ & & & $2.03(1.05)$ \\
\hline
\end{tabular}

scaled to the proportion of root and rhizome biomass present in each plant.

Daily growth rates ( $g$ FW [fresh weight] $d^{-1}$ ) were converted to $\mu \mathrm{mol} \mathrm{Cd^{-1 }}$ required for plant growth $\left(G_{\mathrm{p}}\right)$ using a ratio of $0.22 \mathrm{~g} \mathrm{DW} \mathrm{g}^{-1} \mathrm{FW}$, and a carbon content of $0.4 \mathrm{~g} \mathrm{C} \mathrm{g} \mathrm{DW}^{-1}$ for new tissue (Alcoverro 1995). The resulting carbon required for growth was added to the metabolic rate calculations in order to determine whole-plant carbon balance $\left(B_{\mathrm{p}}\right)$ :

$$
B_{\mathrm{p}}=C_{\text {gain }}-\left(D_{\mathrm{R}-\mathrm{R}}+C_{\mathrm{p}}\right)
$$

Positive values of $B_{\mathrm{p}}$ indicate that daily integrated photosynthesis fixed more carbon than was required to meet the daily demand of respiration and growth, leading to the accumulation of stored carbon reserves. Negative values of $B_{\mathrm{p}}$ indicate that photosynthesis was insufficient to offset daily carbon demand, requiring the mobilization of stored carbon. 


\section{RESULTS}

\section{Metabolic rates and leaf chlorophyll}

$P_{\mathrm{m}}$ decreased significantly from Leaf 2 to 3 to 4 (Tables $1 \& 2$, Tukey test $p<0.05$ for all pairwise comparisons of leaves). The mean photosynthetic capacity of Leaf 2 was $132 \%$ of Leaf 3 and $192 \%$ of Leaf 4 . Respiration rates, in contrast, were not affected by leaf age (Tables $1 \& 2$ ). As a result of the differences in $P_{\mathrm{m}}$, the instantaneous $P_{\text {gross }}: R$ of Leaf 2 was 3.71 , Leaf 3 was 3.50 and Leaf 4 was 2.39. Chlorophyll content of leaves within each age category declined significantly over time (Table 1, Fig. 2). Additionally, $P_{\mathrm{m}}$ declined significantly along the axis of Leaf 2 from 5 freshly collected plants (Fig. 3, ANOVA, $F=7.41, p<0.003$ ). Rates of respiration again showed no significant age-dependent effects within the leaf (ANOVA, $F=1.46, p=0.26$ ). Respiration rates of the youngest (Leaf 1 ) rhizome internode and root bundle were statistically identical and showed no significant effects of Time or $H_{\text {sat }}$ (Tables $2 \& 3$ ).

\section{Total carbohydrate reserves}

Soluble sugar represented more than $95 \%$ of the TCR in all tissues sampled during the experiment, with the

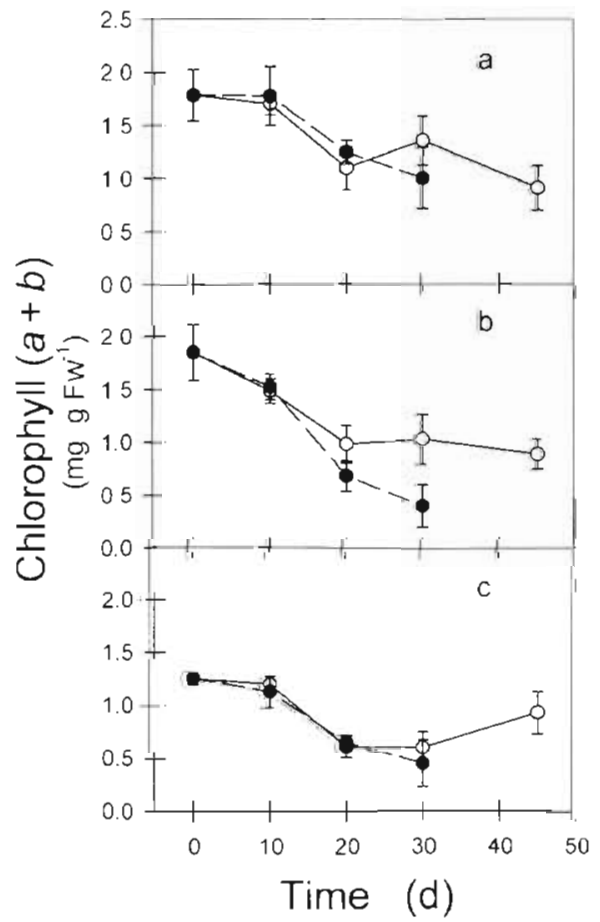

Fig. 2. Temporal changes in chlorophyll $(a+b)$ content measured on middle segments of (a) Leaf 2, (b) Leaf 3 and (c) Leaf 4 growing under $2(\bullet)$ and $7 \mathrm{~h}(0) H_{\mathrm{sal}}$ periods. Error bars indicate standard error of 5 replicate measures. FW: fresh weight

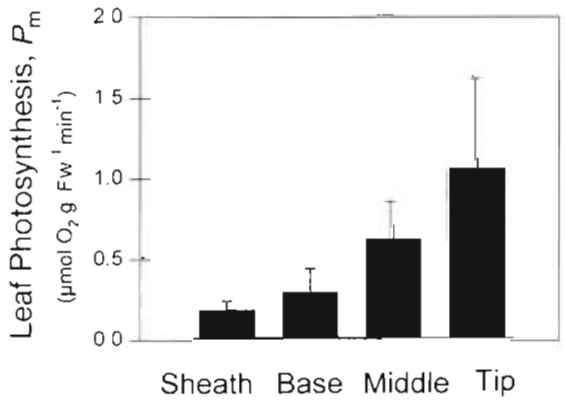

Fig. 3. Rates of light-saturated photosynthesis $\left(P_{\mathrm{m}}\right)$ in different portions of Leaf 2 . Leaves were divided into sheath (that portion below the abscission line), base (the lower third of the leaf above the abscission line), middle (middle third of the leaf) and tip (upper third of the leaf). Error bars indicate standard error

remaining $5 \%$ consisting of starch. TCR decreased through time in both treatments, but there was no significant effect of $H_{\text {sat }}$ or the interaction term (Time $x$ $H_{\text {sat }}$ ) on leaf TCR (Fig. 4, Table 4). Almost $70 \%$ of the TCR initially present in the leaves was depleted in both $H_{\text {sat }}$ treatments over the course of the experiment. Rates of rhizome TCR depletion were internode dependent. TCR concentrations in the youngest internode (Internode 1) declined at equivalent rates in both that were equivalent in both $H_{\text {sat }}$ treatments (Fig. 5, Table 3). TCR levels in Internode 3 declined by almost $50 \%$ over $30 \mathrm{~d}$ in the $2 \mathrm{~h}$ treatment, but remained unchanged in the $7 \mathrm{~h}$ treatment (Fig. 5, Table 3). There was no significant

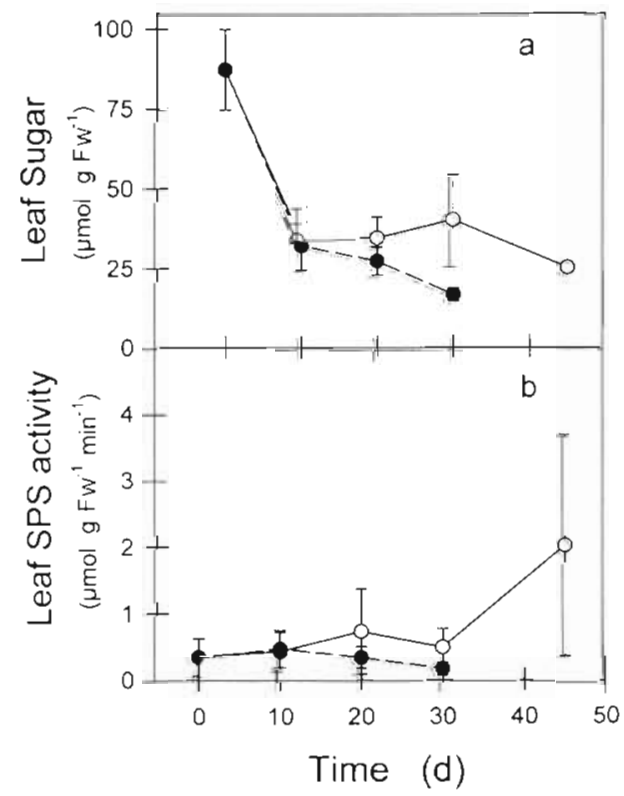

Fig. 4. Temporal changes in (a) sugar content of leaf No. 3 and (b) activity of SPS of Leaf 3 growing under $2(\bullet)$ and $7 \mathrm{~h}(0)$ $H_{\text {sat }}$ periods. Error bars indicate standard error of 5 replicate measures 
Table 3. Results of 2-way ANOVA testing the effects of Time and $H_{\text {sat }}$ on rhizome biomass, root biomass, rhizome respiration (Internode 1), rhizome sugar content (Internodes 1, 3 and 6), root respiration, root sugar content, root SS and root protein. See Table 1 for explanations. (•) $p<0.01$

\begin{tabular}{|c|c|c|c|c|c|c|}
\hline Dependent variable & Independent variable & $\mathrm{df}$ & MS & $F$ & $\mathrm{p}$ & Significance \\
\hline $\begin{array}{l}\text { Rhizome biomass } \\
\text { (g FW) }\end{array}$ & $\begin{array}{l}\text { Time } \\
H_{\text {sat }} \\
\text { Time } \times H_{\text {sat }} \\
\text { Within }\end{array}$ & $\begin{array}{r}3 \\
1 \\
3 \\
35\end{array}$ & $\begin{array}{l}3.15 \\
0.24 \\
0.18 \\
1.81\end{array}$ & $\begin{array}{l}1.73 \\
0.13 \\
0.09\end{array}$ & $\begin{array}{l}0.176 \\
0.713 \\
0.959\end{array}$ & $\begin{array}{l}\text { ns } \\
\text { ns } \\
\text { ns }\end{array}$ \\
\hline $\begin{array}{l}\text { Root biomass } \\
\text { (g FW) }\end{array}$ & $\begin{array}{l}\text { Time } \\
H_{\text {sat }} \\
\text { Time } \times H_{\text {sat }} \\
\text { Within }\end{array}$ & $\begin{array}{r}3 \\
1 \\
3 \\
35\end{array}$ & $\begin{array}{l}0.32 \\
1.27 \\
0.87 \\
0.70\end{array}$ & $\begin{array}{l}0.46 \\
1.81 \\
1.23\end{array}$ & $\begin{array}{l}0.715 \\
0.186 \\
0.312\end{array}$ & $\begin{array}{l}\text { ns } \\
\text { ns } \\
\text { ns }\end{array}$ \\
\hline $\begin{array}{l}\text { Internode } 1 \text { resp } \\
\left(\mu \mathrm{mol} \mathrm{O}_{2} \mathrm{~g}^{-1} \mathrm{FW} \mathrm{min}^{-1}\right)\end{array}$ & $\begin{array}{l}\text { Time } \\
H_{\text {sat }} \\
\text { Time } \times H_{\text {sdt }} \\
\text { Within }\end{array}$ & $\begin{array}{r}3 \\
1 \\
3 \\
35\end{array}$ & $\begin{array}{l}0.0003 \\
5 \times 10^{-6} \\
0.0002 \\
0.0005\end{array}$ & $\begin{array}{l}0.53 \\
0.01 \\
0.47\end{array}$ & $\begin{array}{l}0.666 \\
0.897 \\
0.711\end{array}$ & $\begin{array}{l}\text { ns } \\
\text { ns } \\
\text { ns }\end{array}$ \\
\hline $\begin{array}{l}\text { Internode } 1 \text { sugar } \\
\left(\mu \mathrm{mol} \mathrm{g}{ }^{-1} \mathrm{FW}\right)\end{array}$ & $\begin{array}{l}\text { Time } \\
H_{\text {sat }} \\
\text { Time } \times H_{\text {sat }} \\
\text { Within }\end{array}$ & $\begin{array}{r}3 \\
1 \\
3 \\
34\end{array}$ & $\begin{array}{c}25774 \\
286 \\
2240 \\
4597\end{array}$ & $\begin{array}{l}5.62 \\
0.06 \\
0.49\end{array}$ & $\begin{array}{l}0.003 \\
0.791 \\
0.696\end{array}$ & $\begin{array}{l}\ddot{*} \\
\text { ns } \\
\text { ns }\end{array}$ \\
\hline $\begin{array}{l}\text { Internode } 3 \text { sugar } \\
\left(\mu \mathrm{mol} \mathrm{g}{ }^{-1} \mathrm{FW}\right)\end{array}$ & $\begin{array}{l}\text { Time } \\
H_{\text {sat }} \\
\text { Time } \times H_{\text {sat }} \\
\text { Within }\end{array}$ & $\begin{array}{r}3 \\
1 \\
3 \\
35\end{array}$ & $\begin{array}{c}13487 \\
23426 \\
7732 \\
4054\end{array}$ & $\begin{array}{l}3.33 \\
5.77 \\
1.91\end{array}$ & $\begin{array}{l}0.03 \\
0.02 \\
0.145\end{array}$ & $\dot{\text { ns }}$ \\
\hline $\begin{array}{l}\text { Internode } 6 \text { sugar } \\
\left(\mu \mathrm{mol} \mathrm{g} \mathrm{g}^{-1} \mathrm{FW}\right)\end{array}$ & $\begin{array}{l}\text { Time } \\
H_{\text {sat }} \\
\text { Time } \times H_{\text {sat }} \\
\text { Within }\end{array}$ & $\begin{array}{r}3 \\
1 \\
3 \\
26\end{array}$ & $\begin{array}{l}23442 \\
1454 \\
1581 \\
7279\end{array}$ & $\begin{array}{l}3.22 \\
0.19 \\
0.22\end{array}$ & $\begin{array}{l}0.039 \\
0.658 \\
0.883\end{array}$ & $\begin{array}{l}\dot{\bullet} \\
\text { ns } \\
\text { ns }\end{array}$ \\
\hline $\begin{array}{l}\text { Root respiration } \\
\left(\mu \mathrm{mol} \mathrm{O} \mathrm{g}^{-1} \mathrm{FW} \mathrm{min}^{-1}\right)\end{array}$ & $\begin{array}{l}\text { Time } \\
H_{\text {sat }} \\
\text { Time } \times H_{\text {sat }} \\
\text { Within }\end{array}$ & $\begin{array}{r}3 \\
1 \\
3 \\
35\end{array}$ & $\begin{array}{c}0.0027 \\
0.0002 \\
0.001 \\
0.0015\end{array}$ & $\begin{array}{l}1.90 \\
0.11 \\
0.72\end{array}$ & $\begin{array}{l}0.146 \\
0.733 \\
0.552\end{array}$ & $\begin{array}{l}\text { ns } \\
\text { ns } \\
\text { ns }\end{array}$ \\
\hline $\begin{array}{l}\text { Root sugar } \\
\left(\mu \mathrm{mol} \mathrm{g}{ }^{-1} \mathrm{FW}\right)\end{array}$ & $\begin{array}{l}\text { Time } \\
H_{\text {sat }} \\
\text { Time } \times H_{\text {sd }} \\
\text { Within }\end{array}$ & $\begin{array}{r}3 \\
1 \\
3 \\
32\end{array}$ & $\begin{array}{l}484.1 \\
0.348 \\
47.11 \\
156.8\end{array}$ & $\begin{array}{l}3.09 \\
0.002 \\
0.3\end{array}$ & $\begin{array}{l}0.04 \\
0.915 \\
0.826\end{array}$ & $\begin{array}{l}\text { ns } \\
\text { ns }\end{array}$ \\
\hline $\begin{array}{l}\text { Root protein } \\
\left(\mathrm{mg} \mathrm{g} \mathrm{g}^{-1} \mathrm{FW}\right)\end{array}$ & $\begin{array}{l}\text { Time } \\
H_{\text {sat }} \\
\text { Time } \times H_{\text {sat }} \\
\text { Within }\end{array}$ & $\begin{array}{r}3 \\
1 \\
3 \\
32\end{array}$ & $\begin{array}{c}0.0013 \\
1.11 \\
3 \\
1\end{array}$ & $\begin{array}{l}0.001 \\
1.11 \\
2.01\end{array}$ & $\begin{array}{l}0.922 \\
0.363 \\
0.08\end{array}$ & $\begin{array}{l}\text { ns } \\
\text { ns } \\
\text { ns }\end{array}$ \\
\hline $\begin{array}{l}\text { Root SS activity } \\
\left(\mu \mathrm{mol} \mathrm{g}^{-1} \mathrm{FW} \mathrm{min}^{-1}\right)\end{array}$ & $\begin{array}{l}\text { Time } \\
H_{\text {sdt }} \\
\text { Time } \times H_{\text {sat }} \\
\text { Within }\end{array}$ & $\begin{array}{r}3 \\
1 \\
3 \\
32\end{array}$ & $\begin{array}{l}2.40 \\
0.56 \\
7.54 \\
2.51\end{array}$ & $\begin{array}{l}0.96 \\
0.22 \\
3\end{array}$ & $\begin{array}{l}0.427 \\
0.645 \\
0.044\end{array}$ & $\begin{array}{l}\text { ns } \\
\text { ns }\end{array}$ \\
\hline
\end{tabular}

effect of $H_{\text {sat }}$ on TCR levels of Internode 6, which declined to $1 / 3$ of its initial level in both treatments (Table 3, Fig 5). Root TCR levels declined at approximately equal rates in both treatments (Fig. 6). Although the effect of Time on root TCR content was statistically significant, there was no effect of $H_{\text {sat }}$ (Table 3 ).

Enzyme activity. There were no significant effects of Time or $H_{\text {sat }}$ on leaf SPS activity for the first $30 \mathrm{~d}$ of the experiment (Fig. 4, Table 4), At 45 d, however, leaf SPS activity of plants exposed to $7 \mathrm{~h} \mathrm{H}_{\text {sat }}$ increased significantly. All the plants grown under $2 \mathrm{~h} H_{\text {sat }}$ died before the $45 \mathrm{~d}$ sampling period. Root SS activity was consistently low in the $2 \mathrm{~h}$ treatment throughout the course of the experiment, but, like SPS activity, rose sharply at $30 \mathrm{~d}$ in the $7 \mathrm{~h} H_{\text {sar }}$ treatment and remained high through the end of the experiment at $45 \mathrm{~d}$ (Fig. 6). The synergistic effect of Time and $H_{\text {sat }}$ on root SS activity is indicated by the statistically significant interaction term in the 2-way ANOVA (Table 3). Leaf and root protein content were not affected by $H_{\text {sat }}$ or time (Tables $3 \& 4$ ).

Growth rates and biomass allocation. Plant biomass, absolute growth rates and shoot-specific growth rates declined in both $2 \mathrm{~h}$ and $7 \mathrm{~h} H_{\text {sat }}$ treatments throughout the $45 \mathrm{~d}$ course of the experiment (Table 4, Fig. 7). In both treatments, the reduction in shoot biomass resulted primarily from loss of the oldest leaf during 
Table 4. Results of 2-way ANOVA examining the effects of Time and $H_{\text {sat }}$ on different variables: shoot biomass, shoot growth rates, leaf carbohydrates, SPS activity and leaf protein. See Table 1 for explanations

\begin{tabular}{|c|c|c|c|c|c|c|}
\hline Dependent variable & Independent variable & $\mathrm{df}$ & MS & $F$ & $\mathrm{p}$ & Significance \\
\hline $\begin{array}{l}\text { Shoot biomass } \\
(\mathrm{g} F W)\end{array}$ & $\begin{array}{l}\text { Time } \\
H_{\text {sat }} \\
\text { Time } \times H_{\text {sat }} \\
\text { Within }\end{array}$ & $\begin{array}{r}3 \\
1 \\
3 \\
32\end{array}$ & $\begin{array}{r}227.4 \\
11.1 \\
3.3 \\
19.2\end{array}$ & $\begin{array}{r}11.82 \\
0.52 \\
0.17\end{array}$ & $\begin{array}{c}<0.001 \\
0.46 \\
0.911\end{array}$ & $\begin{array}{l}\cdots \\
\mathrm{ns} \\
\mathrm{ns}\end{array}$ \\
\hline $\begin{array}{l}\text { Shoot growth } \\
\left(\mathrm{cm} \mathrm{d}^{-1}\right)\end{array}$ & $\begin{array}{l}\text { Time } \\
H_{\text {sat }} \\
\text { Time } \times H_{\text {sat }} \\
\text { Within }\end{array}$ & $\begin{array}{r}2 \\
1 \\
2 \\
24\end{array}$ & $\begin{array}{r}84.2 \\
109.4 \\
12.3 \\
23.1\end{array}$ & $\begin{array}{l}3.65 \\
4.75 \\
0.53\end{array}$ & $\begin{array}{l}0.040 \\
0.037 \\
0.597\end{array}$ & $\dot{\text { ns }}$ \\
\hline $\begin{array}{l}\text { Specific shoot growth } \\
\left(\mathrm{d}^{-1}\right)\end{array}$ & $\begin{array}{l}\text { Time } \\
H_{\text {sat }} \\
\text { Time } \times H_{\text {sat }} \\
\text { Within }\end{array}$ & $\begin{array}{r}2 \\
1 \\
2 \\
25\end{array}$ & $\begin{array}{l}0.59 \\
0.70 \\
0.08 \\
0.11\end{array}$ & $\begin{array}{l}5.38 \\
6.34 \\
0.75\end{array}$ & $\begin{array}{l}0.011 \\
0.018 \\
0.484\end{array}$ & $\dot{:}$ \\
\hline $\begin{array}{l}\text { Carbohydrates } \\
\left(\mu \mathrm{mol} \text { suc } \mathrm{g}^{-1} \mathrm{FW}\right)\end{array}$ & $\begin{array}{l}\text { Time } \\
H_{\text {sat }} \\
\text { Time } \times H_{\text {sat }} \\
\text { Within }\end{array}$ & $\begin{array}{r}3 \\
1 \\
3 \\
34\end{array}$ & $\begin{array}{r}1706 \\
707 \\
275 \\
445\end{array}$ & $\begin{array}{r}20.76 \\
1.58 \\
0.61\end{array}$ & $\begin{array}{r}<0.001 \\
0.214 \\
0.611\end{array}$ & $\begin{array}{l}\cdots \\
\text { ns } \\
\text { ns }\end{array}$ \\
\hline $\begin{array}{l}\text { SPS activity } \\
\left(\mu \text { mol suc } \mathrm{g}^{-1} \mathrm{FW} \mathrm{min}^{-1}\right)\end{array}$ & $\begin{array}{l}\text { Time } \\
H_{\text {sat }} \\
\text { Time } \times H_{\text {sat }} \\
\text { Within }\end{array}$ & $\begin{array}{r}3 \\
1 \\
3 \\
28\end{array}$ & $\begin{array}{l}0.094 \\
0.032 \\
0.090 \\
0.045\end{array}$ & $\begin{array}{l}2.08 \\
0.70 \\
1.99\end{array}$ & $\begin{array}{l}0.125 \\
0.41 \\
0.14\end{array}$ & $\begin{array}{l}\text { ns } \\
\text { ns } \\
\text { ns }\end{array}$ \\
\hline $\begin{array}{l}\text { Leaf protein } \\
\left(\mathrm{mg} \mathrm{g}^{-1} \mathrm{FW}\right)\end{array}$ & $\begin{array}{l}\text { Time } \\
H_{\text {sal }} \\
\text { Time } \times H_{\text {sat }} \\
\text { Within }\end{array}$ & $\begin{array}{r}3 \\
1 \\
3 \\
27\end{array}$ & $\begin{array}{l}1.19 \\
0.30 \\
6.42 \\
5.94\end{array}$ & $\begin{array}{l}0.20 \\
0.05 \\
1.08\end{array}$ & $\begin{array}{l}0.89 \\
0.81 \\
0.37\end{array}$ & $\begin{array}{l}\text { ns } \\
\text { ns } \\
\text { ns }\end{array}$ \\
\hline
\end{tabular}

the first week of the experiment. By Day 20, growth rates of the $2 \mathrm{~h}$ plants were significantly lower than the $7 \mathrm{~h}$ plants, and biomass was significantly lower by Day 30 (Fig. 7, Table 4). In total, 7 of the 25 plants grown under $2 \mathrm{~h} H_{\text {sat }}$ treatment died within the first $30 \mathrm{~d}$, before they could be harvested for analysis. None of the $2 \mathrm{~h}$ plants survived to be sampled at $45 \mathrm{~d}$. In contrast, only 2 of 25 plants from the $7 \mathrm{~h} H_{\text {sat }}$ treatment died before the experiment was terminated at $45 \mathrm{~d}$, despite similar TCR concentrations and depletion rates. Plants produced new leaves (between 0 and 1) throughout the course of the experiment, but new roots were not initiated in either treatment. Plants in both treatments were devoid of roots on the first 2 to 3 nodes, and existing roots were generally long, fibrous and gray rather than the bright yellow to white colors typical of new rapidly growing roots.

\section{Whole plant carbon balance}

Whole plant carbon budgets calculated using only the middle section of each leaf showed a strong linear relationship to the more detailed calculation integrating different leaf segments $\left(r^{2}=0.97\right)$. The significant differences in $P_{\mathrm{m}}$ associated with leaf position and age (Fig. 3), however, resulted in a consistent under-estimate of the carbon budget in this case (slope $=1.41 \pm$

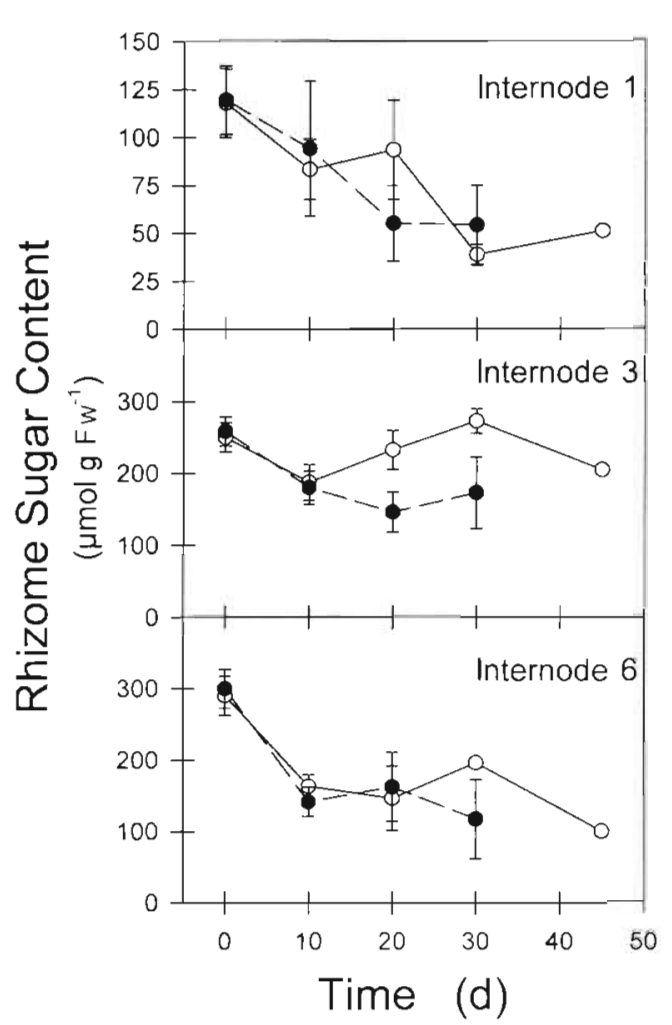

Fig. 5. Temporal changes in rhizome sugar content of Internodes 1,3 and 6 growing under $2(\bullet)$ and $7 \mathrm{~h}(0) H_{\text {sat }}$ periods. Error bars indicate standard error of 5 replicate measures 


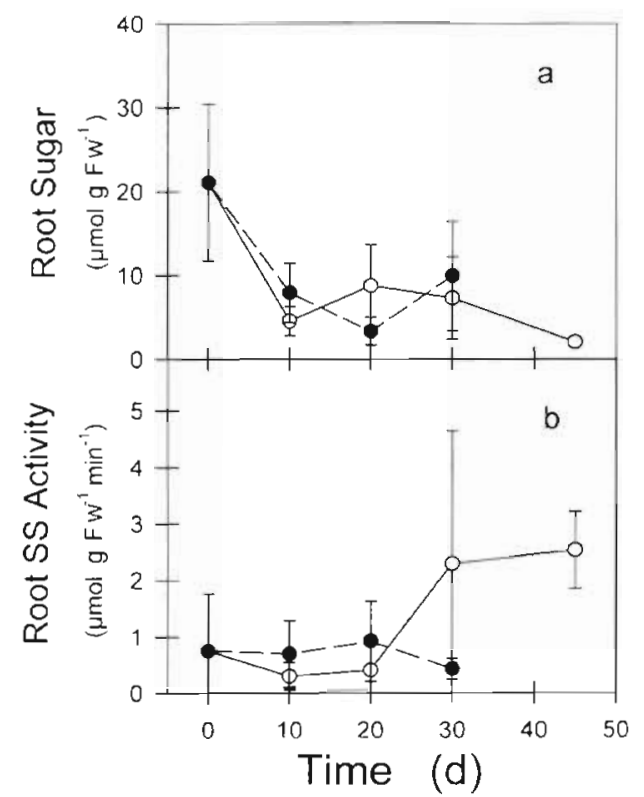

Fig. 6. (a) Temporal changes in root sugar content (first bundle) growing under $2(\bullet)$ and $7 \mathrm{~h}(0) H_{\text {sat }}$ periods. (b) Temporal changes in SS activity in the roots (first bundle) growing under 2 (-) and $7 \mathrm{~h}(0) H_{\text {sat }}$ periods. Error bars indicate standard error of 5 replicate measures

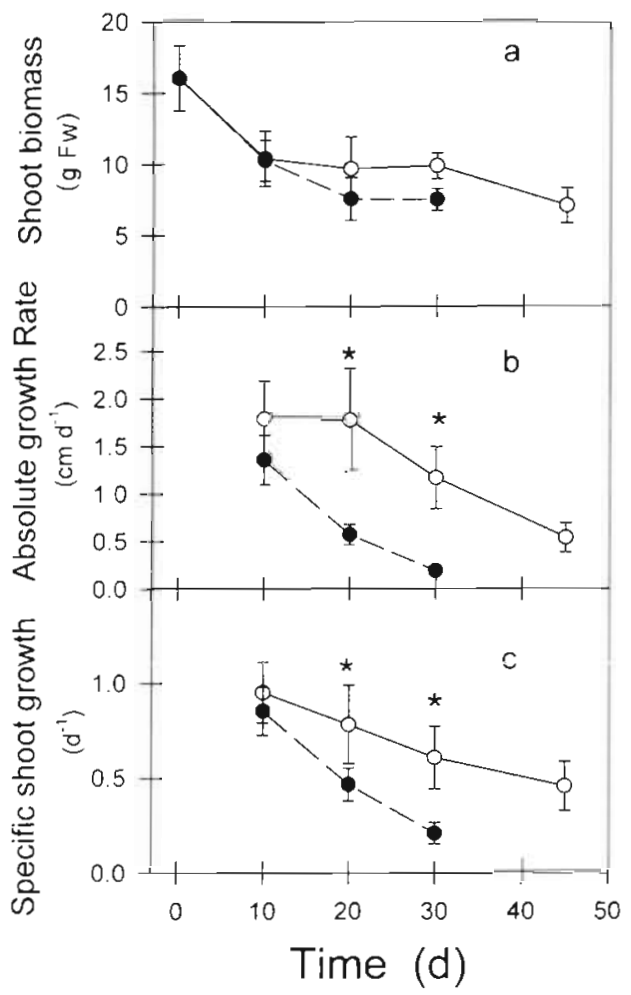

Fig. 7. Temporal changes in (a) shoot biomass, (b) shoot growth rates and (c) specific shoot growth growing under 2 $(\bullet)$ and $7 \mathrm{~h}(\mathrm{O}) H_{\mathrm{sat}}$ periods. Error bars indicate standard error of 5 replicate measures. ( $\star$ ) Values significantly different from zero $(p<0.05, t$-test $)$

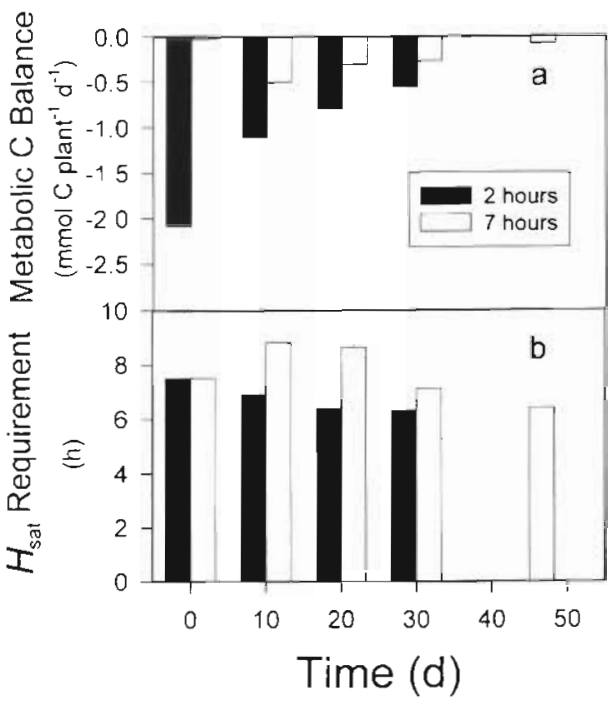

Fig. 8. (a) Estimates of daily metabolic carbon balance for plants growing under 2 and $7 \mathrm{~h} H_{\text {sat }}$ during the course of the experiment. (b) Estimated $H_{\text {sat }}$ periods required to meet daily carbon demand, based on metabolic carbon balance calcula tions for plants growing under 2 and $7 \mathrm{~h} H_{\text {sot }}$

$0.12, t$-test for difference from slope $=1: t=11.7, \mathrm{df}=8$, $p<0.0001, y$-intercept $=162 \pm 92, t$-test for differences from $y$-intercept $=0: t=-1.12, \mathrm{p}=0.30$ ). Consequently, the carbon balance estimates for the $H_{\text {sat }}$ experiment were multiplied by 1.41 to correct for this difference caused by the high variation in leaf $P_{\mathrm{m}}$

Although plants maintained under $2 \mathrm{~h} H_{\text {sat }}$ were more severely carbon limited than plants grown under $7 \mathrm{~h} H_{\text {sat }}$ whole-plant carbon balance calculated from the metabolic and growth rates was negative for both $H_{\text {sat }}$ treatments throughout the course of this experiment (Fig. 8). Thus, growth and survival in both $H_{\text {sat }}$ treatments required the mobilization of stored reserves to balance carbon demand. Metabolic carbon demand calculated from rates of phoiosynthesis, respiration and growth was able to account for almost all (79 \pm $26 \%$ ) the carbon depletion observed in both treatments (Fig. 9, $r^{2}=0.57$, slope $=-0.79 \pm 0.26$, $t$-test for difference from slope $=1: t=-2.98, \mathrm{df}=6, \mathrm{p}=0.03, y$ intercept $=-196 \pm 139, t$-test for differences from $y$ intercept $=0: t=-1.42, \mathrm{p}=0.21$ ). Given that these calculations did not include carbon lost by the sloughing of senescent older leaves in the first $10 \mathrm{~d}$ (roughly $20 \%$ of the shoot biomass), observed rates of carbon depletion and calculated rates based on metabolic demand appear to be essentially in balance. Carbon balance became less negative through time as plant size and growth rates of both treatments declined, but the $H_{\text {sat }}$ period required to meet that demand remained relatively constant at about $7.4 \mathrm{~h}$ because metabolic rates remained constant (Fig. 8 ). 


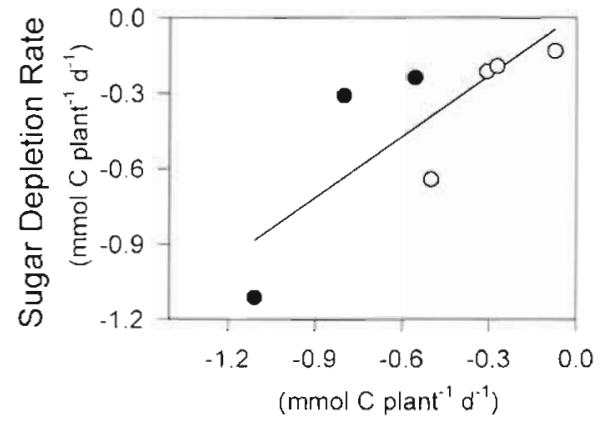

Metabolic Carbon Demand

Fig. 9. Relationship between observed rates of sugar depletion and daily carbon balance calculated from the sum of metabolic rates and growth for plants under $2(\bullet)$ and $7 \mathrm{~h}(0)$

$H_{\text {sat }}$. Regression line was fit to the combined data set

\section{DISCUSSION}

Internal carbon reserves clearly provide an important buffer to extend eelgrass survival when photosynthesis is inadequate to maintain positive daily carbon balance. This mobilization of carbohydrate reserves accumulated during periods of abundant light availability represents an important strategy for survival of perennial seagrasses in temporally variable environments, and appears to be considerably more common than other strategies that include seasonal dormancy and annual life histories exhibited by populations found at the extremes ranges of eelgrass distribution (Keedy \& Patriquin 1978, Gagnon et al. 1980, Phillips and Backman 1983, Robertson \& Mann 1984, Harrison 1993). Although low rates of photosynthesis resulted in negative carbon balances for both treatments throughout this study, the $2 \mathrm{~h} H_{\text {sat }}$ treatment imposed a severely short period of daily photosynthesis and belowground aerobiosis that led to plant death within $30 \mathrm{~d}$ despite consuming only $2 / 3$ of the carbohydrate reserves stored in rhizomes of the dead plants. This inablity to fully mobilize below-ground reserves demonstratesthat translocation was perhaps disrupted in the $2 \mathrm{~h}$ plants by the extended night time anoxia, as has been shown in a short-term experiment (Zimmerman \& Alberte 1996). Thus, severe light limitation can lead to plant death before below-ground carbon reserves are completely exhausted.

Rates of TCR utilization by leaves, roots, and rhizomes observed here were consistent with the carbon demand calculated from growth and metabolism of tissue segments. Quantitative agreement between these approaches underscores the reliability of whole-plant carbon balance estimates derived from careful respirometry of leaf, rhizome and root segments when the light environment is carefully characterized or controlled (Zimmerman et al. 1995b, 1996, Zimmerman \& Mobley
1997). Discrepancies between laboratory-based calculations and in situ respirometry of whole plants have been reported, particularly in turbid environments that produce large gradients in light availability through the canopy (Herzka \& Dunton 1997). Unless these light gradients are measured and modeled accurately using radiative transfer theory, laboratory photosynthesis versus irradiance models are likely to over-estimate production if irradiance is assumed to be uniform over the entire canopy (Zimmerman \& Mobley 1997). The in situ measures, however, are likely to produce site and eventspecific correlations that are difficult to generalize to other periods, locations or seagrass populations.

The patterns of TCR depletion in leaves, roots and rhizomes provide insight into the buffering capacity of reserve mobilization in response to light-availability. The decline in shoot growth that began after $10 \mathrm{~d}$ was clearly a response to overall carbon balance, as has been observed in Thalassia testudinum and Posidonia oceanica (Tomasko \& Dawes 1989, Alcoverro 1995). Continued leaf elongation and proliferation of new leaves up to the point of plant death in the absence of any root proliferation also demonstrates that the photosynthetic shoot is the primary sink for reduced carbon at the expense of the roots. Release from carbon limitation, however, results in the rapid re-direction of photosynthate into the roots of eelgrass (Zimmerman et al. 1996).

Total carbohydrate reserves of Internode 3 were least affected by the severe reduction in light availability imposed by the $2 \mathrm{~h} H_{\text {sat }}$ treatment, indicating that this internode acts as an important conduit for the transport of carbon reserves from older internodes to the meristem. Although TCR declined monotonically in Internodes 1 and 6 in both $H_{\text {sat }}$ treatments, Internode 3 TCR declined significantly only in the $2 \mathrm{~h} H_{\text {sat }}$ treatment, and then only after $20 \mathrm{~d}$. Furthermore, plants in the $2 \mathrm{~h} H_{\text {sat }}$ treatment died with more than $100 \mu \mathrm{mol}$ sucrose equivalent $\mathrm{g}^{-1} \mathrm{FW}$ remaining in Internode 3 , roughly $75 \%$ of the TCR present in this internode at the beginning of the experiment. In contrast, TCR levels in Internodes 1 and 6 dropped almost 3-fold during the course of this experiment. The $2 \mathrm{~h} H_{\text {sat }}$ plants died shortly after equilibration of the sucrose gradient between Internodes 6 and 3 even though $1 / 3$ of the TCR present at the beginning of the experiment remained unutilized. Thus, eelgrass may require a strong source-sink gradient to maintain adequate delivery of reduced carbon to the meristem when translocation is limited to short daily periods of aerobiosis.

The lack of healthy root growth even during the initial phase of this experiment indicates that negative carbon balance inhibits root production on new internodes of eelgrass, as it does in a variety of terrestrial plants (Pierson et al. 1990, Sims \& Pearcy 1994). The 
lack of new roots on the first 3 internode segments at the first sampling period revealed that plants probably had been carbon-limited at the time of collection. Thus, the proliferation of metabolically active roots may occur only when whole-plant carbon balance is positive (Zimmerman et al. 1996). The lack of roots, however, may provide eelgrass with a less secure hold on the sediment, leaving plants vulnerable to physical disturbance and erosion.

The final phase of carbon depletion was characterized by cessation of growth and changes in enzyme activity. The increased activity of root SS in the $7 \mathrm{~h} H_{\text {sat }}$ plants at $30 \mathrm{~d}$ may represent a stress response to increase the sink strength of severely carbon limited tissues, as has been described previously in eelgrass and maize in response to anoxia (Freeling \& Bennett 1985, McCarty et al. 1986, Xue et al. 1991, Zimmerman et al. 1995b, 1996). Leaf SPS activity, which controls sucrose loading from photosynthetic sources (Huber et al. 1985), is unresponsive to shifts in light availability or photosynthetic rate in carbon-replete eelgrass, unlike many terrestrial plant species (Zimmerman et al. 1995a). However, the significant increase in SPS activity reported here for $7 \mathrm{~h} H_{\text {sat }}$ plants at $45 \mathrm{~d}$ may provide another indicator of severe carbon stress.

In contrast to the observed changes in carbon reserves and growth rates, metabolic rates were insensitive to the temporal increases in carbon limitation imposed by both light regimes in this study. Rates of $P_{\mathrm{m}}$ from both $7 \mathrm{~h}$ and $2 \mathrm{~h} H_{\text {sat }}$ treatments were 30 to $50 \%$ lower than previously reported for Zostera marina, but leaf $R$ remained consistent with the literature (Dennison \& Alberte 1986, Zimmerman et al. 1989, 1991). Rates of root respiration were lower than previously published values. Respiration rates of healthy young roots are typically about $50 \%$ of leaf $R$ and $200 \%$ of rhizome $R$, but decline significantly with age (Zimmerman et al, 1989, Kraemer \& Alberte 1993). Thus, the low rates of root respiration observed here probably reflect the metabolic activity of older roots and the lack of new root production in these carbon-limited plants.

The decline in photosynthetic capacity with age in leaf tissue also represents a significant contrast with earlier reports which showed relatively little variation in light-saturated photosynthetic capacity as a function of leaf age and had allowed the construction of accurate carbon budgets from measures performed on a few leaf segments (Zimmerman et al. 1995a, 1996, Zimmerman \& Mobley 1997). In addition, the rates of $P_{\mathrm{m}}$ reported here were considerably lower than previously published results for Zostera marina from the same population and other locations (Dennison \& Alberte 1995, Zimmerman et al. 1989, 1991, 1995a,b, 1996). The plants in this study, however, were heavily grazed by Tectura depicta. These effects are most evident in older leaves that have been grazed for longer periods of time and correlate well with a reduction in leaf chlorophyll content (Zimmerman et al. 1996). Such variations must be considered whenever calculating whole-plant metabolic carbon balances, as was done here by increasing the number and range of leaf segments measured.

Although the $7 \mathrm{~h}$ treatment provided a realistic winter $H_{\text {sat }}$ period, it was not sufficient to maintain positive carbon balance in these plants that required about

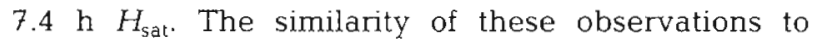
freshly collected plants from the field (Zimmerman unpubl. data) indicates that the experimental conditions did not significantly alter daily carbon requirements of the plants or their metabolic capacity for carbon assimilation relative to plants growing in situ. Clearly, winter conditions in temperate ecosystems can lead to negative carbon balance in eelgrass, especially when plants are stressed by other factors such as leaf grazing or high water column turbidity. The eelgrass studied here responded to negative carbon balances by suppressing the production of new roots, depleting of sucrose reserves, and effecting a slow decline in growth rate and an increase in SS activity in sink tissues in the terminal stages of carbon stress. These patterns may provide useful indices for assessing the state and fate of seagrass ecosystems in advance of catastrophic declines.

Acknowledgements. Many thanks to James Coyer, Olga Invers and Diana Steller for help with the field work. Carlos Duarte, Jorge Terrados and Rohan Arthur generously provided critical comments that significantly improved the manuscript. Financial support was provided by a CIRIT (Comissionat per a Universitats i Recerca)Training Grant to T.A. and by grant OCE-9223265 from the US National Science Foundation to R.C.Z. and R.S.A.

\section{LITERATURE CITED}

Alcoverro T (1995) Production ecology of the Mediterranean seagrass Posidonia oceanica (L) Delile. PhD thesis, University of Barcelona

Backman TW, Barilotti DC (1976) Irradiance reduction: effects on standing crops of the eelgrass Zostera marina in a coastal lagoon. Mar Biol 34:33-40

Canham C, Berkowitz A, Kelly V, Lovett G, Ollinger S, Schnurr J (1996) Biomass allocation and multiple limitation in tree seedlings. Can J For Res 26:1521-1530

Carruthers T, Walker D (1997) Light climate and energy flow in the seagrass canopy of Amphibolis griffithi (JM Black) den Hartog. Oecologia 109:335-341

Clabby G, Osborne B (1997) Irradiance and nitrate-dependent variation in growth and biomass allocation in Mycelis muralis. An analysis of its significance for a functional categorization of 'sun' and 'shade' plants. New Phytol 135:539-547

Dennison WC, Alberte RS (1982) Photosynthetic responses of Zostera marina L. (eelgrass) to in situ manipulations of light intensity. Oecologia 55:137-144

Dennison WC, Alberte RS (1985) Role of daily light period in the depth distribution of Zostera marina (eelgrass). Mar Ecol Prog Ser 25:51-61 
Dennison WC, Alberte RS (1986) Photoadaptation and growth of Zostera marina L. (eelgrass) transplants along a depth gradient. J Exp Mar Biol Ecol 98:265-282

Duarte CM (1991) Seagrass depth limits. Aquat Bot 40:363-377

Dunton KH, Tomasko DA (1994) In situ photosynthesis in the seagrass Halodule wrightii in a hypersaline subtropical lagoon. Mar Ecol Prog Ser 107:281-293

Fettweis M, Sas M, Monbaliu J (1998) Seasonal, neap-spring and tidal variation of cohesive sediment concentration in the Scheldt Estuary, Belgium. Estuar Coast Shelf Sci 47: $21-36$

Freeling M, Bennett DC (1985) Maize Adh1. Annu Rev Genet 19:297-323

Gagnon PS, Vadas RL, Burdick DB, May B (1980) Genetic identity of annual and perennial forms of Zostera marina L. Aquat Bot 8:157-162

Harlin MM, Thorne-Miller B (1981) Nutrient enrichment of seagrass beds in a Rhode-Island coastal lagoon. Mar Biol 65:221-229

Harrison P (1993) Variations in demography of Zostera marina and $Z$. noltii on an intertidal gradient. Aquat Bot 45:63-77

Herzka S, Dunton K (1997) Seasonal photosynthetic patterns in the seagrass Thalassia testudinum in the western Gulf of Mexico. Mar Ecol Prog Ser 152:103-117

Hillman K, Mccomb AJ, Walker DI (1995) The distribution, biomass and primary production of the seagrass Halophila ovalis in the Swan-Canning Estuary, Western Australia. Aquat Bot 51:1-54

Huber SC, Israel DW (1982) Biochemical basis for partitioning of photosynthetically fixed carbon between starch and sucrose in soybean (Glycine max Merr.) leaves. Plant Physiol 69:691-696

Huber SC, Kerr PS, Kalt-Torres W (1985) Regulation of sucrose formation and movement. In: Heath RL, Preiss J (eds) Regulation of carbon partitioning in photosynthetic tissues. Proc 8th Symp Am Soc Plant Physiol, Rockville, MD, p 199-215

Iizumi $H_{1}$ Hattori A (1982) Growth and organic production of eelgrass (Zostera marina L.) in temperature waters of the Pacific Coast of Japan. III: the kinetics of nitrogen uptake. Aquat Bot 12:245-265

Jeffrey SW, Humphrey GF (1975) New spectrophotometric equations for the determination of chlorophylls $a, b, c_{1}$ and $c_{2}$ in higher plants, algae and natural phytoplankton. Biochem Physiol Pflanz 167:191-194

Keedy CJ, Patriquin DG (1978) An annual form of seagrass in Nova Scotia. Aquat Bot 5:163-170

Kraemer GP, Alberte RS (1993) Age-related patterns of metabolism and biomass in subterranean tissues of Zostera marina (eelgrass). Mar Ecol Prog Ser 95:193-203

McCarty DR, Shaw JR, Hannah LC (1986) The cloning, genetic mapping and expression of the constitutive sucrose synthase locus of maize. Proc Natl Acad Sci USA 83:9099-9103

McRoy CP (1969) Eelgrass under Artic winter ice. Nature 224: $818-819$

Moore KA, Wetzel RL, Orth RJ (1997) Seasonal pulses of turbidity and their relations to eelgrass (Zostera marina $\mathrm{L}$.) survival in an estuary. J Exp Mar Biol Ecol 215:115-134.

Pennock JR, Sharp JH (1994) Temporal alternation between light- and nutrient-limitation of phytoplankton production in a coastal plain estuary. Mar Ecol Prog Ser 111:275-288

Perez M, Duarte CM, Romero J, Sand-Jensen K, Alcoverro T (1994) Growth plasticity in Cymodocea nodosa stands: the importance of nutrient supply. Aquat Bot 47:249-264
Phillips R, Backman T (1983) Phonology and reproductive biology of eelgrass (Zostera marina L.) at Bahia Kino, Sea of Cortez, Mexico. Aquat Bot 17:85-90

Pierson E, Mack R, Black R (1990) The effect of shading on photosynthesis, growth and regrowth following defoliation for Bromus tectorum. Oecologia 84:534-543

Pirc H (1989) Seasonal changes in soluble carbohydrates, starch, and energy content in Mediterranean seagrasses. PSZN I: Mar Ecol 10:97-105

Robertson A, Mann K (1984) Disturbance by ice and life-history adaptations of eelgrass Zostera marina. Mar Biol 80:131-141

Sims D, Pearcy R (1994) Scaling sun and shade photosynthetic acclimation of Alocasia macrorrhiza to whole-plant performance: I. Carbon balance and allocation at different daily photon flux densities. Plant Cell Environ 17:881-887

Smith RD (1989) Anaerobic metabolism in the roots of the seagrass Zostera marina L. PhD thesis, The University of Chicago

Tomasko DA, Dawes CJ (1989) Evidence for physiological integration between shaded and unshaded short shoots of Thalassia testudinum. Mar Ecol Prog Ser 54:299-305

Winterbourne DJ (1986) Cell growth determined by a dyebinding assay. Biochem Soc Trans 14:1179

Xue ZT, Larson K, Jochimsen BU (1991) Oxygen regulation of uricase and sucrose synthase synthesis in soybean callus tissue is exerted at the mRNA level. Plant Mol Biol 16: $899-906$

Yemn EW, Willis AJ (1954) The estimation of carbohydrates in plant extracts by anthrone. Biochem J 57:508-514

Zieman JC (1974) Methods for the study of the growth and production of turtle grass, Thalassia testudinum König. Aquaculture 4:139-143

Zimmermann RC, Alberte RS (1996) Effect of light/dark transition on carbon translocation in eelgrass Zostera marina seedlings. Mar Ecol Prog Ser 136:305-309

Zimmerman $\mathrm{RC}$, Mobley $\mathrm{C}$ (1997) Radiative transfer within seagrass canopies: impact on carbon budgets and light requirements. Ocean Optics XIII Proc, SPIE 2963:331-336

Zimmerman RC, Smith RD, Alberte RS (1987) Is growth of eelgrass nitrogen limited? A numerical simulation of the effects of light and nitrogen on the growth dynamics of Zostera marina. Mar Ecol Prog Ser 41:167-176

Zimmerman RC, Smith RD, Alberte RS (1989) Thermal acclimation and whole-plant carbon balance in Zostera marina L. (eelgrass). J Exp Mar Biol Ecol 130:93-109

Zimmerman RC, Reguzzoni JL, Wyllie-Echeverria S, Josselyn M, Alberte RS (1991) Assessment of environmental suitability for growth of Zostera marina L. (eelgrass) in San Franscisco Bay. Aquat Bot 39:353-366

Zimmerman RC, Cabello-Pasini A, Alberte RS (1994) Modeling daily production of aquatic macrophytes from irradiance measurements: a comparative analysis. Mar Ecol Prog Ser 114:185-196

Zimmerman RC, Kohrs DG, Steller DL, Alberte RS (1995a) Carbon partitioning in eelgrass: regulation by photosynthesis and the response to daily light-dark cycles. Plant Physiol 108:1665-1671

Zimmerman RC, Reguzzoni JL, Alberte RS (1995b) Eelgrass (Zostera marina L.) transplants in San Francisco Bay: role of light availability on metabolism, growth and survival. Aquat Bot 51:67-86

Zimmerman RC, Kohrs DG, Alberte RS (1996) Top-down impact through a bottom-up mechanism: the effect of limpet grazing on growth, productivity and carbon allocation of Zostera marina L. (eelgrass). Oecologia 107:560-567

Submitted: January 13, 1999; Accepted: May 28, 1999

Proofs received from author(s): October 5, 1999 